05,12

\title{
Фазовые переходы и фазовые превращения в нанообластях фазового расслоения в мультиферроике $\mathrm{ErMn}_{2} \mathrm{O}_{5}$
}

\author{
(C) Б.Х. Ханнанов, В.А. Санина , Е.И. Головенчиц, С.Г. Лушников \\ Физико-технический институт им. А.Ф. Иофрфе РАН, \\ Санкт-Петербург, Россия \\ "E-mail: sanina@mail.ioffe.ru
}

Поступила в Редакцию 24 июня 2021 г.

В окончательной редакции 24 июня 2021 г.

Принята к публикации 27 июня 2021 г.

В мультиферроике $\mathrm{ErMn}_{2} \mathrm{O}_{5}$ изучено влияние редкоземельного иона $\mathrm{Er}^{3+}$, обладающего большим орбитальным вкладом в магнитный момент, на фазовые переходы и состояния 2D-нанообластей фазового расслоения. Эти области представляют собой полупроводниковые гетероструктуры и формируются за счет процессов самоорганизации в матрице $\mathrm{ErMn}_{2} \mathrm{O}_{5}$. Обнаружено существенное влияние ионов $\mathrm{Er}^{3+}$, моменты которых жестко ориентированы вдоль оси с кристалла, на магнитную динамику, теплоемкость и мультиферроидные свойства слоев гетероструктур в широком интервале температур 5-300 K.

Ключевые слова: мультиферроик, 2D-нанообласти фазового расслоения,полупроводниковые гетероструктуры, фазовые переходы, фазовые превращения.

DOI: 10.21883/FTT.2021.11.51589.155

\section{1. Введение}

Кристаллы $R \mathrm{Mn}_{2} \mathrm{O}_{5}(R$ - редкоземельные ионы, $\mathrm{Bi})$ относятся к мультиферроикам II-го типа, в которых при температурах ниже температуры Кюри $\left(T_{\mathrm{C}} \approx 30-35 \mathrm{~K}\right)$ возникает сегнетоэлектрическое упорядочение, индуцированное магнитным упорядочением с температурой Нееля $\left(T_{\mathrm{N}} \approx 35-40 \mathrm{~K}\right)$ [1,2]. При комнатной температуре они описываются центросимметричной пр.гр. Pbam [3]. Благодаря близости температур магнитного и сегнетоэлектрического упорядочений такие мультиферроики обладают сильным магнитоэлектрическим эффектом.

Характерной особенностью $R \mathrm{Mn}_{2} \mathrm{O}_{5}$ является наличие равного количества ионов $\mathrm{Mn}^{3+}$ (содержащих три $t_{2 g}$ и один $e_{g}$ электрон на $3 d$-оболочке) и $\mathrm{Mn}^{4+}$ (с тремя $t_{2 g}$ электронами на $3 d$-оболочке), что обеспечивает условия для появления диэлектрического зарядового упорядочения. Ионы $\mathrm{Mn}^{4+}$ имеют октаэдрическое кислородное окружение и расположены в слоях с $z=0.25 c$ и $(1-z)=0.75 c$. Связанные между собой цепочки октаэдров с ионами $\mathrm{Mn}^{4+}$ вытянуты вдоль оси $c(z)$. Ионы $\mathrm{Mn}^{3+}$ имеют нецентральное локальное окружение в виде пятиугольных пирамид в слоях с $z=0.5 c$. Эти пирамиды расположены в плоскостях $x y$ и связывают цепочки октаэдров с ионами $\mathrm{Mn}^{4+}$. Ионы $R^{3+}$ с окружением подобным $\mathrm{Mn}^{3+}$ находятся в слоях с $z=0$ [3]. Структура $R \mathrm{Mn}_{2} \mathrm{O}_{5}$ представлена на рис. 1 .

Зарядовое упорядочение и конечная вероятность переноса $e_{g}$ электронов между парами ионов $\mathrm{Mn}^{3+}-\mathrm{Mn}^{4+}$ (двойной обмен $[4,5]$ ) являются ключевыми факторами, определяющими мультиферроидные свойства $R \mathrm{Mn}_{2} \mathrm{O}_{5}$ при всех температурах.
Низкотемпературное сегнетоэлектрическое состояние при $T \leq T_{\mathrm{C}}$ преимущественно обусловлено зарядовым упорядочением вдоль оси $b$. Чередование пар соседних ионов $\mathrm{Mn}^{3+}$ и $\mathrm{Mn}^{4+}$ вдоль оси $b$ с сильным ферромагнитным (двойным) обменом и слабым косвенным антиферромагнитным обменом приводит к обменной стрикции, нарушающей центросимметричность решетки вдоль оси $b$ и появлению низкотемпературного сегнетоэлектрического упорядочения [6]. Перенос же $e_{g}$ электронов

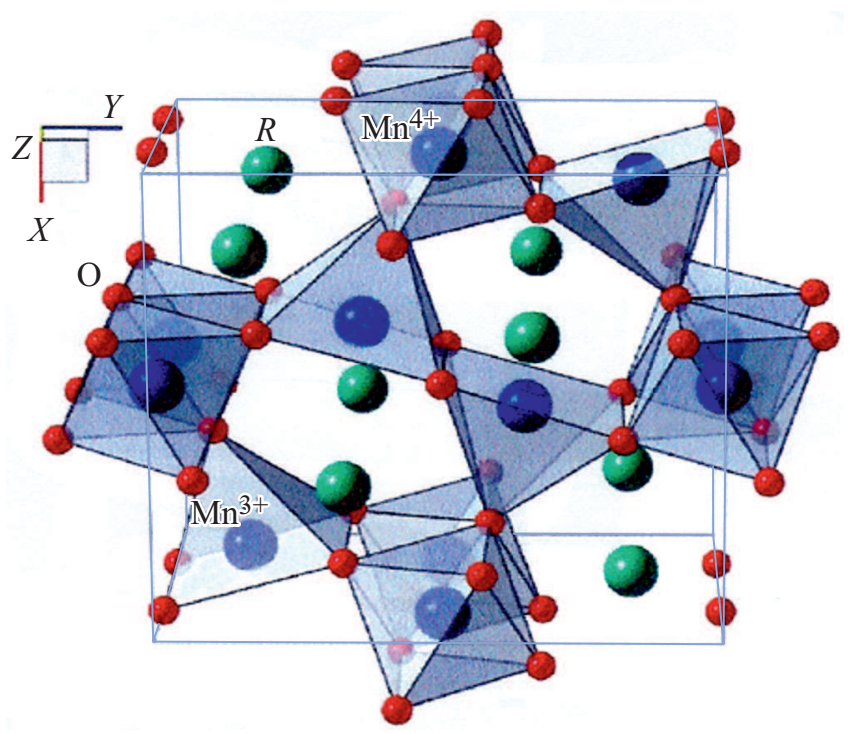

Рис. 1. Структура $R \mathrm{Mn}_{2} \mathrm{O}_{5}$. Малые красные шарики - ионы кислорода; Синего цвета шары - ионы $\mathrm{Mn}^{4+}$ (в октаэдрах) и $\mathrm{Mn}^{3+}$ (в пятиугольных пирамидах). Указаны направления осей кристалла. 


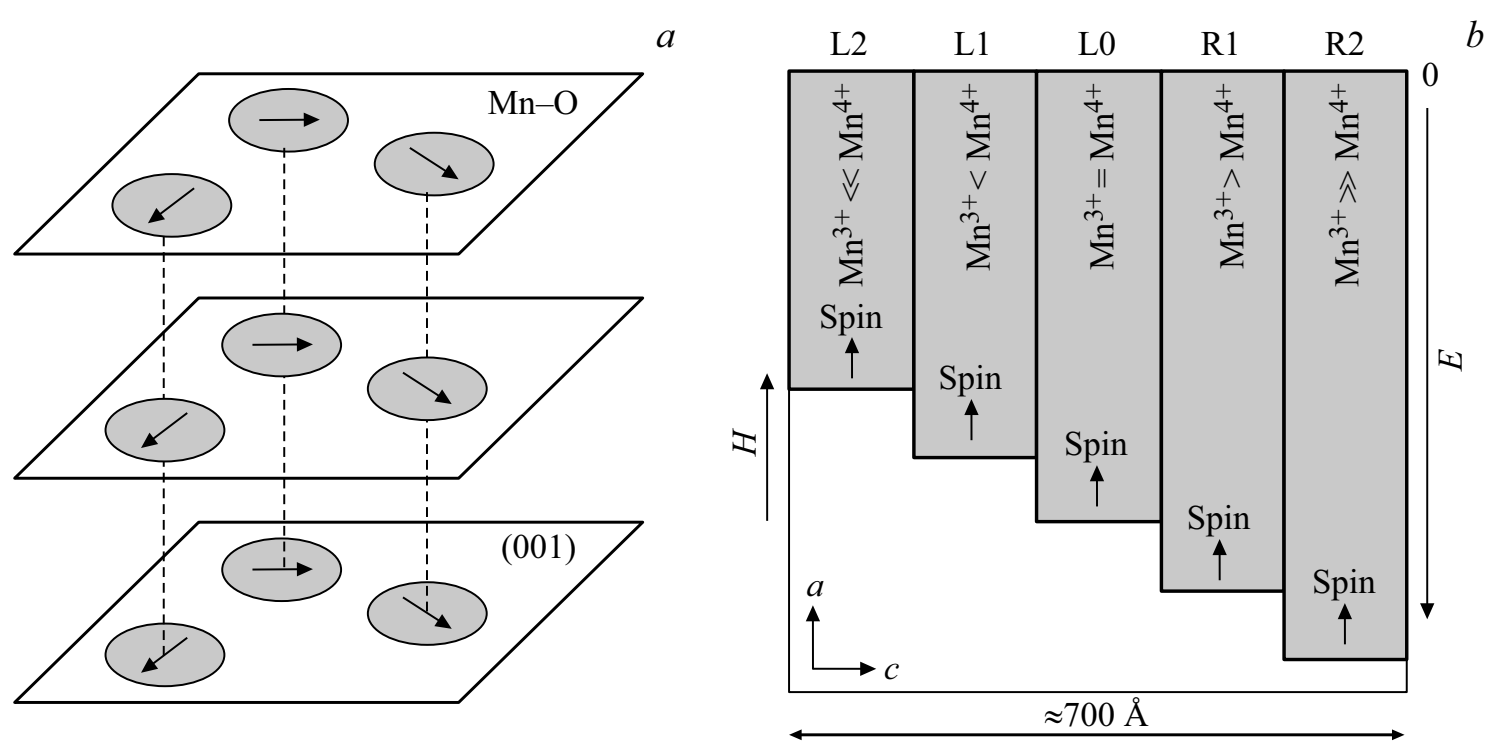

Рис. 2. Схематическое изображение ферромагнитных 1D-сверхрешеток (залитые области), расположенных в исходной матрице кристалла (белое поле), поле $H=0(a)$. Схематическое изображение одной из подобных сверхрешеток в ЕСМО, состоящих из $\mathrm{L}_{\mathrm{N}}$ ферромагнитных слоев, перпендикулярных оси $c$, с различными концентрациями пар ионов $\mathrm{Mn}^{3+}-\mathrm{Mn}^{4+}$ и $e_{g}$ электронов, расположенных в ямах различной глубины (залитые области, с различной энергией $E)(b)$.

между парами ионов $\mathrm{Mn}^{3+}-\mathrm{Mn}^{4+}$, расположенными в соседних слоях, перпендикулярных оси $c$, приводит к образованию нанообластей фазового расслоения с иным распределением ионов $\mathrm{Mn}^{3+}$ и $\mathrm{Mn}^{4+}$ по сравнению с исходной матрицей кристалла. Такие локальные области в as grown образцах являются одновременно магнитными и сегнетоэлектрическими. Они существуют от низких температур до температур, значительно превышающих температуры мультиферроидного упорядочения. При этом сильное магнитоэлекрическое взаимодействие в областях фазового расслоения может существовать до высоких температур.

Состояния областей фазового расслоения были исследованы в мультиферроиках $R \mathrm{Mn}_{2} \mathrm{O}_{5}$, а также в легированных $R_{0.8} \mathrm{Ce}_{0.2} \mathrm{Mn}_{2} \mathrm{O}_{5}(R=\mathrm{Eu}, \mathrm{Gd}, \mathrm{Tb}, \mathrm{Bi})$ [7-21]. В них проводились исследования диэлектрических и магнитных свойств, теплоемкости, рентгеновской дифракции, рамановского рассеяния света [7-13], электрической поляризации [14-19], $\mu \mathrm{SR}$-исследования [20,21]. Отметим при этом, что матрицы кристаллов $R \mathrm{Mn}_{2} \mathrm{O}_{5}$ и легированных $R_{0.8} \mathrm{Ce}_{0.2} \mathrm{Mn}_{2} \mathrm{O}_{5}$ имели одинаковую центральную симметрию Pbam.

Области фазового расслоения формируются в $R \mathrm{Mn}_{2} \mathrm{O}_{5} \quad$ и $R_{0.8} \mathrm{Ce}_{0.2} \mathrm{Mn}_{2} \mathrm{O}_{5}$ аналогично манганитам $\mathrm{La} A M n O_{3}(A=\mathrm{Sr}, \mathrm{Ca}, \mathrm{Ba})$, также содержащим ионы $\mathrm{Mn}^{3+}$ и $\mathrm{Mn}^{4+}$ [22-24]. Они образуются в матрице исходного кристалла в результате процессов самоорганизации, обусловленных конечной вероятностью туннелирования $e_{g}$ электронов между парами ионов $\mathrm{Mn}^{3+}-\mathrm{Mn}^{4+}$ в соседних плоскостях, перпендикулярных оси $c$. Как и в $\mathrm{La} A M n O_{3}(A=\mathrm{Sr}, \mathrm{Ca}, \mathrm{Ba})$, это происходит при балансе сильных взаимодействий: двойного обмена (с характерной энергией $0.3 \mathrm{eV})$, взаимодействия Яна-Теллера
$(0.7 \mathrm{eV})$ и кулоновского отталкивания $(1 \mathrm{eV})$. По этой причине они существуют в широком диапазоне температур от низких до температур выше комнатной [8-19]. Первые два взаимодействия способствуют накоплению электронов и пар ионов $\mathrm{Mn}^{3+}-\mathrm{Mn}^{4+}$ в областях фазового расслоения, кулоновское отталкивание обеспечивает равновесную концентрацию электронов и форму этих областей [22-24]. Слоистая структура с распределением ионов $\mathrm{Mn}^{3+}$ и $\mathrm{Mn}^{4+}$ в слоях, перпендикулярных оси $c$, в основной матрице кристаллов $R \mathrm{Mn}_{2} \mathrm{O}_{5}$ и $R_{0.8} \mathrm{Ce}_{0.2} \mathrm{Mn}_{2} \mathrm{O}_{5}$ сохраняется и в областях фазового расслоения. В результате, области фазового расслоения формируют полупроводниковые гетероструктуры, содержащие различное количество ионов $\mathrm{Mn}^{3+}$ (являющихся донорами) и ионов $\mathrm{Mn}^{4+}$ (являющихся акцепторами) в слоях сверхрешеток. Эти гетероструктуры могут быть представлены в виде периодически изменяющихся изотропных ферромагнитных слоев с ферромагнитными же границами между ними, не препятствующими переносу $e_{g}$ электронов между слоями при двойном обмене. Наиболее подробно свойства таких гетероструктур изучены в $\mathrm{EuMn}_{2} \mathrm{O}_{5}(\mathrm{EMO})$ и $\mathrm{Eu}_{0.8} \mathrm{Ce}_{0.2} \mathrm{Mn}_{2} \mathrm{O}_{5}$ (ECMO) [8-12].

На рис. 2 для примера схематически показаны способ формирования ферромагнитных полупроводниковых гетероструктур (1D-сверхрешеток) (рис. 2,a), а также изображение одной из подобных сверхрешеток в ЕСМО (рис. 2,b) [25]. От отдельных слоев сверхрешеток наблюдался набор ферромагнитных резонансов (ФМР), особенности которых позволяли судить о свойствах этих слоев и всей сверхрешетки в целом. Интенсивность и положение по магнитному полю отдельных линий ФМР сверхрешеток определяются различным количеством ферромагнитных пар ионов $\mathrm{Mn}^{3+}$ и $\mathrm{Mn}^{4+}$ в 
слоях сверхрешеток. В ЕМО и ЕСМО 1D-сверхрешетки существуют до температур $T<60 \mathrm{~K}$ и $T<80 \mathrm{~K}$ соответственно. Размеры областей фазового расслоения в них $\approx 900 \AA \quad($ ЕМО) и $\approx 700 \AA,($ ЕСМО) $[8]$.

Баланс сильных взаимодействий, указанных выше, обеспечивает формирование динамически равновесных состояний областей фазового расслоения, при которых имеется определенное распределение ионов $\mathrm{Mn}^{3+}$ и $\mathrm{Mn}^{4+}$ в отдельных слоях сверхрешеток.

Основной задачей исследований в настоящей работе является изучение влияния на свойства $1 \mathrm{D}-$-верхрешеток областей фазового расслоения в $\mathrm{ErMn}_{2} \mathrm{O}_{5}$ (ErMO) ионов $\mathrm{Er}^{3+}$, основное состояние которого качественно отличается от состояния иона $\mathrm{Eu}^{3+}$ в ЕСМО. Ионы $\mathrm{Eu}^{3+}$ в основном состоянии ${ }^{7} F_{0}$ являются практически немагнитными. Это так называемые ван-флековские ионы, для которых характерно слабое подмешивание первого возбужденного магнитного состояния ${ }^{7} F_{1}$, удаленного от основного состояния на $\approx 300 \mathrm{~cm}^{-1}$ и практически не влияющее на свойства ЕСМО. Основное состояние иона $\mathrm{Er}^{3+}\left({ }^{4} I_{15 / 2}, S=3 / 2, L=6\right)$ обладает большим магнитным моментом $\left(J=9.6 \mu_{\mathrm{B}}\right)$, в который основной вклад вносит орбитальный момент и возникает сильное кристаллическое поле, которое жестко ориентирует моменты ионов $\mathrm{Er}$ вдоль оси $c$, индуцируя сильную одноионную анизотропию. В результате в ErMO возникают добавочные взаимодействия по сравнению с ЕСМО, формирующие иное распределение пар ионов $\mathrm{Mn}^{3+}-\mathrm{Mn}^{4+}$ в слоях сверхрешеток. Зарядовое упорядочение при этом сохраняется (равенство концентраций ионов $\mathrm{Mn}^{3+}-\mathrm{Mn}^{4+}$ во всей сверхрешетке диктуется сильным электростатическим взаимодействием и сохранением заряда). Таким образом, в ErMO и ECMO различаются как величины обменного магнитного взаимодействия $R$ ионов с подсистемой ионов марганца, так и анизотропия этого взаимодействия. Спины пар ионов $\mathrm{Mn}^{3+}-\mathrm{Mn}^{4+}$ в ErMO жестко ориентированы вдоль оси $c$, в то время как в ЕСМО они сравнительно легко ориентируются вдоль направления приложенного внешнего магнитного поля $[10,11]$.

В настоящей работе мы провели сравнительное исследование магнитных свойств, теплоемкости, магнитной динамики (набора ферромагнитных резонансовот слоев сверхрешеток) и электрической поляризации $\mathrm{ErMO}$. Особое внимание было уделено особенностям свойств областей фазового расслоения в $\mathrm{ErMnO}$. Выявлены согласующиеся друг с другом в измерениях различными методами особенности свойств ErMO, обусловленные ионами $\mathrm{Er}^{3+}$.

\section{2. Объекты и методы исследования}

Монокристаллы ErMO выращивались методом спонтанной кристаллизации из раствора-расплава [26,27]. Они имели форму пластинок толщиной $2-3 \mathrm{~mm}$ и площадью $3-5 \mathrm{~mm}^{2}$. Симметрия кристаллов и их состав определялись рентгеновским фазовым анализом и рентгеновским флюоресцентным методом, соответственно. Все кристаллы имели пластинчатую огранку с хорошо определяемыми осями. Ось $c$ обычно была перпендикулярна развитой плоскости пластинок, и четко определялись ориентации осей $a$ и $b$. Для измерений использовались монокристаллы ErMO с естественной огранкой.

Измерялась динамическая магнитная восприимчивость $\mathrm{ErMO}$ индукционным методом на частоте $10 \mathrm{kHz}$. Образец выбранной ориентации, помещался в одну из двух предварительно скомпенсированных измерительных катушек и измерялся сигнал раскомпенсации, вызванный магнитным моментом образца.

Для измерений ФМР использовался спектрометр магнитного резонанса проходного типа с малой магнитной модуляцией. Измерения проводились в диапазоне температур $5-300 \mathrm{~K}$, на частотах $30-40 \mathrm{GHz}$ в магнитном поле до $2 \mathrm{~T}$, создаваемом электромагнитом. Криостат с оптическими окнами помещался в микроволновый канал, обеспечивающий однородное распределение СВЧ-волны вблизи образца. Постоянное магнитное поле $H$ перпендикулярно направлению распространения микроволнового поля (волнового вектора $k$ ). Детектированные сигналы ФМР усиливались SR530 Lock-in усилителем.

Для измерения теплоемкости использовался универсальный прибор для измерения физических свойств PPMS „Quantum Design“.

Для измерения диэлектрических свойств и поляризации изготавливались плоские конденсаторы толщиной $0.3-0.6 \mathrm{~mm}$ и площадью $3-4 \mathrm{~mm}^{2}$. Диэлектрическая проницаемость и проводимость определялись с помощью измерителя импеданса Good Will LCR-819 в частотном интервале $0.5-50 \mathrm{kHz}$.

Электрическая поляризация измерялась PUND (Positive Up Negative Down) методом. Нами использовался адаптированный к измерениям поляризации локальных полярных областей с локальной проводимостью метод, описанный в $[15,16,18]$.

\section{3. Экспериментальные данные и их анализ}

\section{1. Магнитная динамика (набор ферромагнитных резонансов от слоев сверхрешеток областей фазового расслоения) в ErMO.}

Как было продемонстрировано при изучении ферромагнитных резонансов (FMR) в ECMO $[9,10]$, pacпределение интенсивностей линий FMR от отдельных слоев областей фазового расслоения позволяет судить о свойствах слоев и всей сверхрешетки в целом. Для выяснения особенностей магнитной динамики в $\mathrm{ErMO}$ удобно провести сравнение этой динамики в ErMO и 

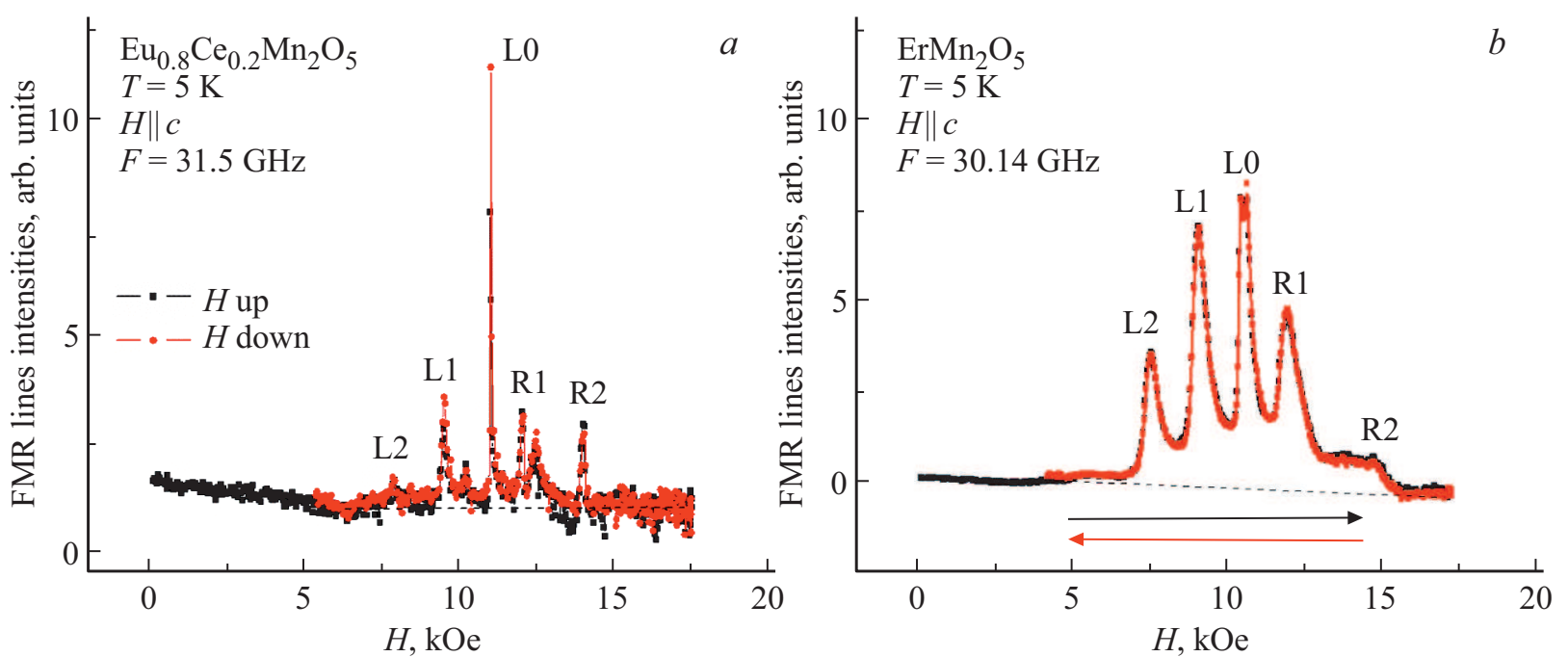

Pис. 3. Наборы линий FMR от слоев сверхрешеток в ЕСMO: $(a)(F=31.5 \mathrm{GHz})$ и $\operatorname{ErMO}(b)(F=30.14 \mathrm{GHz}) . H \| c . T=5 \mathrm{~K}$.

ЕСМО. Как отмечалось выше, ионы $\mathrm{Eu}^{3+}$ в основном состоянии являются практически немагнитными и слабо связанными с решеткой. В то время как ионы $\mathrm{Er}^{3+}$, напротив, являются сильно магнитными и сильно искажают решетку. На рис. $3, a$ и $b$ представлены наборы FMR от слоев сверхрешеток в ЕСМО и ЕrMO соответственно.

Динамически равновесное состояние слоев сверхрешеток в ЕСМО, изображенное на рис. 3, $a$, устанавливалось после последовательных трех циклирований магнитного поля (его нарастаний и снижений) при измерении FMR.

При этом циклировании постепенно изменялись состояния слоев сверхрешеток, причем после каждого цикла состояния оказывались достаточно долгоживущими, чтобы происходило накопление последовательных изменений состояний от цикла к циклу. Время протяжки магнитного поля в одну из сторон (при скорости $1.2 \mathrm{kOe} / \mathrm{min}$ ) составляло $\sim 15 \mathrm{~min}$. Интенсивности сигналов FMR от отдельных слоев сверхрешеток пропорциональны их намагниченности, т.е. числу ферромагнитных пар ионов $\mathrm{Mn}^{3+}$ и $\mathrm{Mn}^{4+}$ в этих слоях. В динамически равновесном состоянии наиболее интенсивной является линия FMR слоя L0, в котором имеется одинаковое количество ионов $\mathrm{Mn}^{3+}$ и $\mathrm{Mn}^{4+}$. В этом слое формируется диэлектрическое состояние с зарядовым упорядочением. В левых слоях L2, L1 число ионов $\mathrm{Mn}^{4+}$ превышает число $\mathrm{Mn}^{3+}$ ионов. Эти слои обладают дырочной проводимостью и интенсивности линий FMR от этих слоев значительно слабее, чем у L0-слоя. В правых слоях R2, R1 число ионов $\mathrm{Mn}^{3+}$ превышает число ионов $\mathrm{Mn}^{4+}$. Эти слои обладают электронной проводимостью и интенсивности линий FMR от этих слоев также значительно слабее, чем у L0-слоя. Таким образом формируется полупроводниковая гетероструктура с зарядовой компенсацией левых и правых слоев относительно L0-слоя. При этом, в случае ЕCMO все линии наблюдаются на нулевом фоне поглощения, показанном штрихами на рис. $3, a$. Схематическое распределение энергетических ям для слоев сверхрешеток в ЕСМО показано на рис. $2, b$.

Качественно иная ситуация возникает с распределением интенсивностей сигналов FMR в ErMO (рис. 3, $b$ ). B ErMO наблюдаются те же пять линий FMR, но эти линии значительно шире и соотношение их интенсивностей существенно иное. Линии не разрешены в своих основаниях, формируя отличный от нуля фон, на котором они наблюдаются. Важным фактом является Tот, что, в отличие от ECMO, полный набор линий FMR в ErMO формируется при первом же цикле изменения магнитного поля, причем ориентированного именно вдоль оси $c$. При ориентациях магнитного поля вдоль осей $a$ и $b$ линий FMR вообще наблюдать не удается.

Полученный в первом цикле приложения поля вдоль оси $c$ набор линий FMR в ErMO не изменяется при следующих циклах изменения поля. Это указывает на то, что в ErMO имеется достаточно сильное внутреннее поле, в котором изначально устанавливается основное состояние сверхрешеток, которое фиксируется уже при первом измерении сигналов FMR. Таким образом, основное динамически равновесное состояние сверхрешеток в ЕrMO формируется самопроизвольно за счет внутренних взаимодействий. В то время как в ЕСМО динамически равновесное (долгоживущее) состояние формируется циклированием магнитного поля. Внутренним взаимодействием, ответственным за это, является взаимодействие ионов $\mathrm{Er}^{3+}$ с ионами $\mathrm{Mn}^{3+}$ и $\mathrm{Mn}^{4+}$. Напомним, что ионы $\mathrm{Er}^{3+}$ обладают большим магнитным моментом, значительную часть которого составляет орбитальный момент, сильно искажающий решетку именно вдоль оси $c$. Вдоль этой же оси в $\mathrm{ErMn}_{2} \mathrm{O}_{5}$ расположены цепочки октаэдров с ионами $\mathrm{Mn}^{4+}$ (рис. 1). Поэтому ионы $\mathrm{Er}^{3+}$ наиболее сильно искажают октаэдры с ионами $\mathrm{Mn}^{4+}$ вдоль оси $c$, углубляя ямы для сло- 


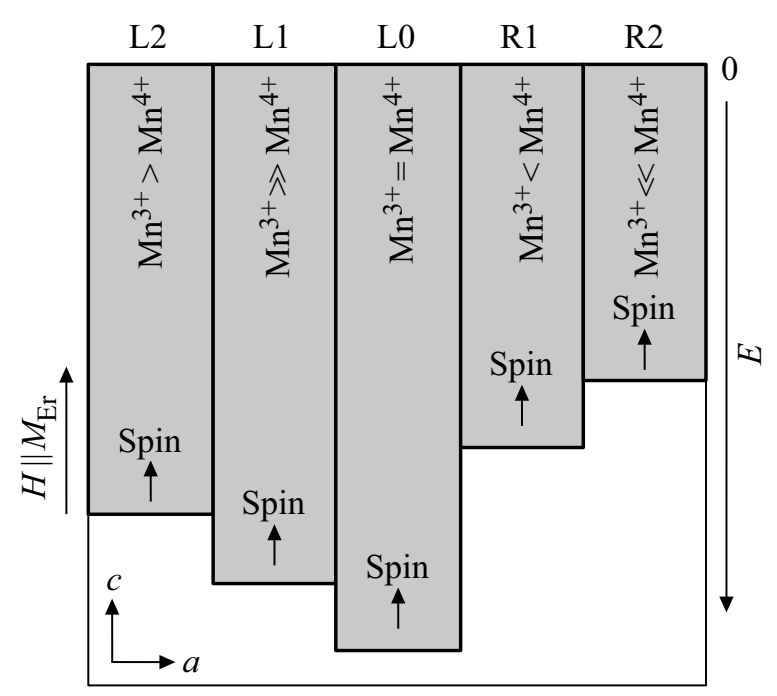

Рис. 4. Схематическое распределение глубин ям слоев сверхрешеток в областях фазового расслоения ErMO.

ев L1 и L2. Это приводит к перетеканию в эти слои электронов из правых слоев L0, R1, R2, увеличивая число ионов $\mathrm{Mn}^{3+}$ и число пар ферромагнитных пар ионов $\mathrm{Mn}^{3+}-\mathrm{Mn}^{4+}$ в слоях L1 и L2 сверхрешеток, формируя новое динамически равновесное состояние во всей сверхрешетке.

Исходя из экспериментально наблюдаемого соотношения интенсивностей линий FMR в ErMO, мы можем схематически представить соотношение глубин ям слоев сверхрешеток и соотношение числа ионов $\mathrm{Mn}^{3+}$ и $\mathrm{Mn}^{4+}$ в отдельных слоях (см. рис. 4). По-прежнему, самым устойчивым и энергетически выгодным является слой LO с зарядовым упорядочением. В нем числа ионов $\mathrm{Mn}^{3+}$ и $\mathrm{Mn}^{4+}$ равны друг другу; возникает диэлектрическое состояние с максимальным числом ферромагнитных пар ионов $\mathrm{Mn}^{3+}-\mathrm{Mn}^{4+}$, что обеспечивает самую интенсивную и узкую линию FMR (рис. $3, b$ ). Глубина ямы L0 максимальна (рис. 4). Как отмечено выше, глубины ям и интенсивности линий FMR слоев L1, L2 в ErMO превышают глубины ям R1, R2 и довольно близки к L0-слою (рис. 4). В более глубокие слои L1, L2 стекают электроны из более мелких слоев, повышая число ян-теллеровских ионов $\mathrm{Mn}^{3+}$ в октаэдрах, за счет реакции $\left(\mathrm{Mn}^{4+}+e=\mathrm{Mn}^{3+}\right)$. Это также увеличивает глубины ям этих слоев. В результате, в ErMO в глубоких слоях L1, L2 преобладают ионы $\mathrm{Mn}^{3+}$, а в слоях R1, $\mathrm{R} 2$ ионы $\mathrm{Mn}^{4+}$ (рис. 4). Т.е. в ErMO наблюдается инвертирование свойств слоев L1, L2 и R1-, R2-слоев по сравнению с ЕСМО (сравни рис. 4 и рис. $2, b$ ).

B ErMO имеется еще одно качественное отличие от ЕСМО. В равновесном состоянии ЕСМО симметричное распределение зарядов в левых и правых слоях относительно L0-линии на нулевом фоне матрицы кристалла приводило к зарядовой компенсации левых и правых слоев сверхрешеток и к отсутствию спонтанной элек- трической поляризации без приложения электрического поля. Как будет продемонстрировано ниже, нарушение симметрии соотношения интенсивностей для линий L1, L2 и R1, R2 относительно L0-линии, а также искажение фона матрицы ErMO, на котором расположены эти линии, свидетельствует о нарушении зарядовой компенсации и появлении электрической поляризации в ErMO.

\section{2. Магнитные свойства $\mathrm{ErMn}_{2} \mathrm{O}_{5}$. Влияние на них областей фазового расслоения.}

На рис. 5, a представлены динамические магнитные восприимчивости вдоль различных осей кристалла ErMO, пропорциональные магнитным моментам образцов в соответствующих направлениях. Так как все кристаллы семейства мультиферроиков $R \mathrm{Mn}_{2} \mathrm{O}_{5}$ с различными редкоземельными ионами, включая и немагнитные ионы $\mathrm{Y}$ and $\mathrm{Bi}$, характеризуются близкими температурами Нееля $T_{\mathrm{N}} \sim 40 \mathrm{~K}$, то можно утверждать, что магнитное упорядочение во всех этих мультиферроиках определяется подсистемой ионов марганца. Магнитное упорядочение ионов $R^{3+}$ в соединениях с $3 d$-ионами (ортотоферритах и ортохромитах) обычно происходит при низких температурах 5-10 K.

B ErMO обусловленный ионами $\mathrm{Er}^{3+}$ резкий рост магнитной восприимчивости наблюдается вдоль оси $c$ при понижении температуры вплоть до $5 \mathrm{~K}$ (рис. 5,a). При этом максимума магнитной восприимчивости, характерного для их магнитного упорядочения не наблюдается. Таким образом, во всем наблюдаемом нами интервале температур от $5 \mathrm{~K}$ и выше ионы $\mathrm{Er}^{3+}$ находятся в парамагнитном состоянии. Вблизи температуры $44 \mathrm{~K}$, соответствующей температуре магнитного упорядочения Мn-подсистемы матрицы кристалла, аномалии восприимчивости вдоль оси $c$ также не наблюдается. Это позволяет предположить, что восприимчивость по оси $c$ определяется преимущественно ионами $\mathrm{Er}^{3+}$.

Парамагнитная восприимчивость ионов $\mathrm{Er}^{3+}$ вдоль оси $c$ при $T>5 \mathrm{~K}$ описывается законом Кюри-Вейсса $\chi=C_{\mathrm{M}} /\left(T-\theta_{p}\right)$ (где $\chi-$ магнитная восприимчивость; $C_{\mathrm{M}}$ - постоянная Кюри, позволяющая определить эффективный магнитный момент; $\theta_{p}$ - температура Кюри, ниже которой возникает спонтанная намагниченность) (рис. 5,b). Как видно, зависимость обратной восприимчивости от температуры линейна в интервале температур 5-65K, хотя наклон $\chi^{-1}(T)$ немного изменяется при $T \approx 25 \mathrm{~K}$. Полученные из закона Кюри-Вейсса значения температуры Кюри и эффективного магнитного момента составляют $\theta_{p}=-4 \mathrm{~K}$ и $\mu \sim 7 \mu \mathrm{B}$ соответственно. Значение эффективного магнитного момента ионов $\mathrm{Er}^{3+}$ оказалось меньше момента изолированного иона $\mathrm{Er}^{3+}(9.6 \mu \mathrm{B})$.

Таким образом, магнитное упорядочение ионов $\mathrm{Er}$ в ErMO может происходить лишь при $T<T_{\mathrm{N} 1} \approx 4 \mathrm{~K}$. При температурах $T>T_{\mathrm{N} 1}$ ионы $\mathrm{Er}$ парамагнитны и ориентируются вдоль оси $c$ внутренним эффективным 

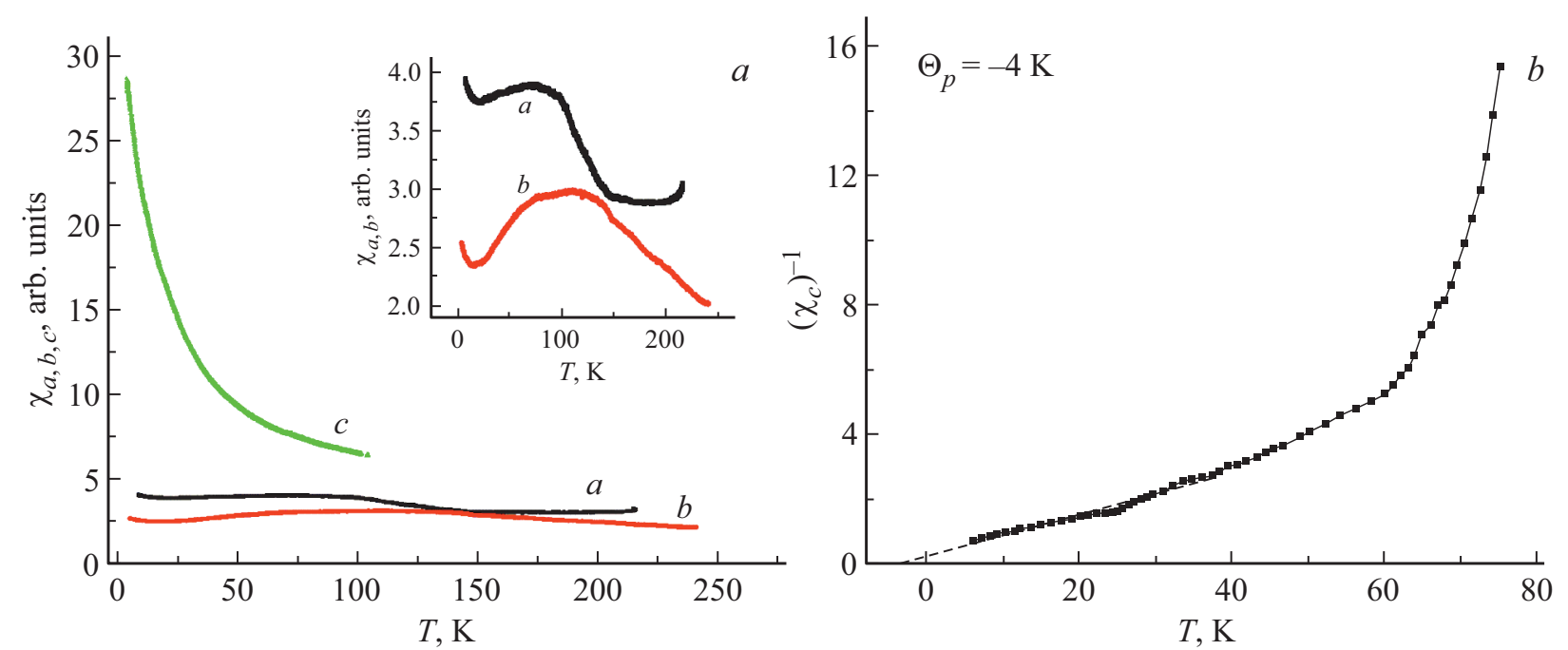

Рис. 5. Зависимости от температур динамической магнитной восприимчивости ErMO вдоль различных осей, указанных у кривых. На вставке: восприимчивости вдоль осей $a$ и $b$ показаны в большем масштабе $(a)$; Зависимость от температуры обратной магнитной восприимчивости ErMO вдоль оси $c(b)$.

полем, обусловленным орбитальным вкладом в $\mathrm{Er}$ момент, а также эффективным полем обменного взаимодействия $\mathrm{Er}-\mathrm{Mn}$. При этом парамагнитная восприимчивость ионов $\mathrm{Er}$ вдоль оси $c$ при $T>T_{\mathrm{N} 1}$ значительно превышает магнитные восприимчивости кристаллов ErMO в направлениях осей $a$ и $b$ (рис. 5, $a$ ). Как отмечалось выше, на ориентацию магнитных моментов $\mathrm{Er}$ также влияет эффективное внутреннее поле $\mathrm{Er}-\mathrm{Mn}$ обмена, зависящее от спиновых ориентаций и величин магнитных моментов ионов $\mathrm{Mn}$.

Выше отмечалось, что магнитные восприимчивости ErMO в плоскости $a b$ (рис. 5, $a$ и вставка к нему) обусловлены преимущественно марганцевыми ионами, расположенными в слоях $a b$ (рис. 1). При этом максимальными магнитными восприимчивостями обладают ферромагнитные пары ионов $\mathrm{Mn}^{3+}-\mathrm{Mn}^{4+}$ в областях фазового расслоения. Тем не менее, как видно из рис. 5, $a$, эти восприимчивости меньше вклада в измеренную восприимчивость вдоль оси $c$, что видимо обусловлено малой концентрацией областей фазового расслоения в объеме кристалла ErMO (также, как и в ЕМО и ЕСМО [7-10]).

На вставке к рис. 5, $a$ в увеличенном масштабе представлены магнитные восприимчивости вдоль осей $a$ и $b$. Видно, что в диапазоне температур 5-25 K наблюдается нарастание восприимчивостей с понижением температуры. В интервале температур 25-65 K нарастание восприимчивостей наблюдается с ростом температуры. Таким образом, вблизи $25 \mathrm{~K}$ происходит смена знака температурного изменения намагниченности марганцевой подсистемы (вставка к рис. $5, a$ ). Вблизи $T \approx 65 \mathrm{~K}$ зависимости $\chi(T)$ вдоль осей $a$ и $b$ выходят на некоторые плато и далее медленно спадают в диапазоне температур $\sim 150-170 \mathrm{~K}$ (в направлении оси $a$ ) и вблизи $250 \mathrm{~K}$ (вдоль оси $b$ ) (вставка к рис. 5, $a$ ). Таким образом, в
ErMO аномальное поведение обратной восприимчивости вблизи $25 \mathrm{~K}$ и довольно резкое изменение наклона ее температурной зависимости вблизи $65 \mathrm{~K}$ (рис. $5, b$ ) указывает на изменение состояния ионов Мn вблизи этих температур.

По отношению к областям фазового расслоения, содержащим ферромагнитные пары ионов $\mathrm{Mn}^{3+}-\mathrm{Mn}^{4+}$ и электроны, перезаряжающие эти ионы в слоях $a b$ при двойном обмене, эффективное магнитное поле ионов $\mathrm{Er}^{3+}$ вдоль оси $c$ оказывается поперечным. Оно вызывает прецессию электронов внутри областей фазового расслоения, индуцируя диамагнитный отклик, понижающий эффективный момент ионов $\operatorname{Er}$ вдоль оси $c$. Это является причиной отмеченного выше уменьшения эффективного магнитного момента ионов $\mathrm{Er}^{3+}$, полученного из закона Кюри-Вейсса для ErMO, по сравнению с его значением для изолированного иона $\mathrm{Er}^{3+}$. Как оказалось, уменьшение момента ионов Er в ErMO также обусловлено влиянием областей фазового расслоения Мn-подсистемы. Таким образом, самосогласованное влияние магнитных Er- и Mn-подсистем друг на друга за счет $\mathrm{Er}-\mathrm{Mn}$ обменного взаимодействия характеризуют температурные корреляции в изменениях состояний $\mathrm{Er}-\mathrm{Mn}$-подсистем. Резкое нарастание намагниченности ионов $\operatorname{Er}$ с понижением температуры усиливает диамагнитный отклик электронов в областях фазового расслоения. Этот отклик, в свою очередь, зависит от концентрации электронов, перезаряжающих ферромагнитные пары ионов $\mathrm{Mn}^{3+}-\mathrm{Mn}^{4+}$ в этих областях, определяя их магнитный момент. Концентрация таких электронов уменьшается с нарастанием поля ионов $\mathrm{Er}$ при понижении температуры до $25 \mathrm{~K}$. При $T<25 \mathrm{~K}$ диамагнитный отклик не увеличивается, а моменты ионов марганца с понижением температуры нарастают по закону Кюри-Вейсса. Описанный процесс определя- 

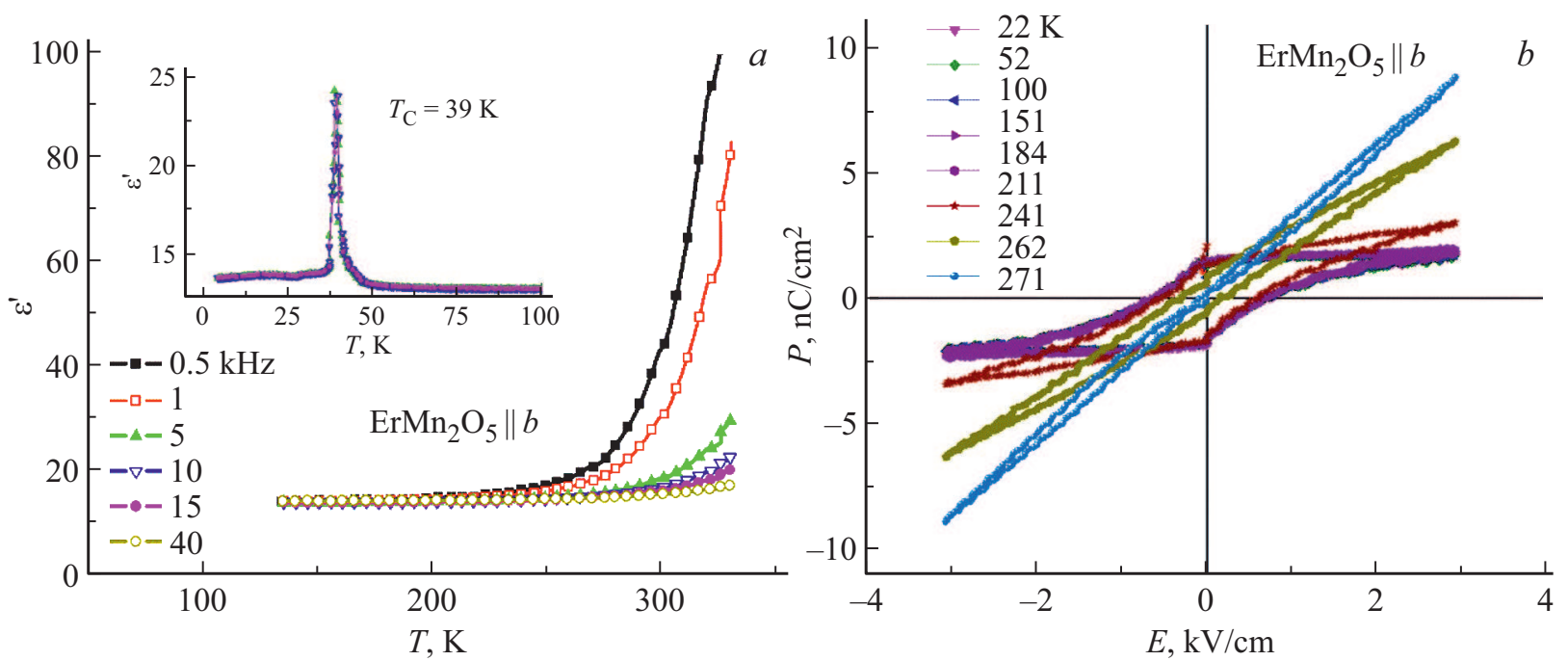

Рис. 6. Температурные зависимости $\varepsilon^{\prime}$ вдоль оси $b$ для ряда частот, указанных на рисунке. На вставке показаны не имеющие частотной дисперсии максимумы $\varepsilon^{\prime}$ для оси $b(a)$; петли электрической поляризации ErMO вдоль оси $b$, измеренные PUND методом для ряда температур $(b)[18]$.

ет поведение намагниченности марганцевой подсистемы ниже $65 \mathrm{~K}$ (вставка к рис. 5,a).

Магнитный вклад областей фазового расслоения ионов Мn вдоль осей $a$ и $b$ при $T>65 \mathrm{~K}$ наиболее отчетливо проявляется при выходе восприимчивостей вдоль этих осей на температурные плато с более медленным их падением при росте температур до указанных выше температур (вставка к рис. 5, a). Эти предельные температуры, при которых происходит падение высокотемпературных магнитных восприимчивостей, естественно связать с температурами, при которых тепловая энергия $k T$ становится равной высоте барьеров на границах областей фазового расслоения и состояния областей фазового расслоения резко изменяются. Ниже этих температур возникает замороженное суперпарамагнитное состояние, характеризующееся наличием магнитных петель гистерезиса [28]. При более высоких температурах области фазового расслоения находятся в обычном суперпарамагнитном состоянии, не обладающем остаточными намагниченностями в магнитном поле, равном нулю.

\section{3. Диэлектрические и полярные свойства ErMO и проявления в них свойств областей фазового расслоения.}

В нашей работе [18] было изучено сегнетоэлектрическое состояние ErMO. На рис. 6, $а$ для определения температуры существования упорядоченного сегнетоэлектрического состояния ErMO представлена температурная зависимость диэлектрической проницаемости вдоль оси $b$ в широком диапазоне температур. На вставке рис. 6, а показаны частотно-независимые максимумы диэлектрической проницаемости $\varepsilon^{\prime}$ вблизи температуры
Кюри $T_{\mathrm{C}} \approx 39 \mathrm{~K}$, свидетельствующие о сегнетоэлектрическом фазовом переходе при этой температуре.

Как было показано в [18], при $T>T_{\mathrm{C}}$ вдоль всех осей ErMO наблюдаются петли гистерезиса с остаточной поляризацией до температур, значительно более высоких чем $T_{\mathrm{C}}$. Мы связывали это с существованием высокотемпературных полярных областей фазового расслоения, формирующих замороженное суперпараэлектрическое состояние, демонстрирующее петли гистерезиса электрической поляризации.

Как видно из рис. 6, $a$ частотно-независимая диэлектрическая проницаемость $\varepsilon^{\prime}$ вдоль оси $b$ минимальна и не зависит от температуры вплоть до $\sim 270 \mathrm{~K}$, начиная с которой начинается частотно-зависимый рост $\varepsilon^{\prime}$. На рис. $6, b$ приведены петли гистерезиса электрической поляризации вдоль оси $b$. Видно, что петли гистерезиса существуют от самых низких температур до температуры, близкой той, при которой начинается резкий рост частотно-зависимых величин диэлектрической проницаемости (рис. 6,a). Аналогичная ситуация имеет место для диэлектрической проницаемости и электрической поляризации вдоль оси $a$ до температуры $\sim 170 \mathrm{~K}$, а вдоль оси $c$ до $\sim 250 \mathrm{~K}$.

На рис. 7 представлены температурные зависимости остаточной поляризации $P_{\text {rem }}$ петель электрической поляризации для всех осей ErMO. Они указывают величины электрических поляризаций для этих осей, а также температуры, до которых такие поляризации существуют. Низкотемпературные аномалии на зависимостях $P_{\text {rem }}$ вдоль оси $b$ при температуре $\sim 39 \mathrm{~K}$ соответствуют низкотемпературному сегнетоэлектрическому переходу матрицы кристалла ErMO (см. вставку к рис. 6,a). Широкая низкотемпературная аномалия $P_{\text {rem }}$ вдоль оси $c$ ниже $\sim 40 \mathrm{~K}$ свидетельствует о структурном изменении 


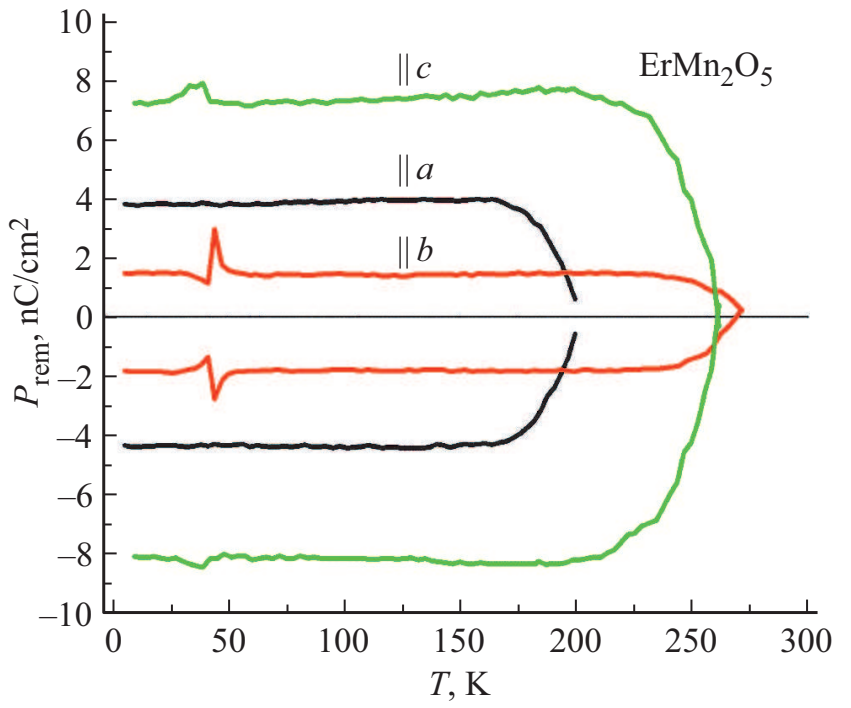

Рис. 7. Температурные зависимости остаточной поляризации $P_{\text {rem }}$ в петлях электрической поляризации, измеренных вдоль всех осей кристалла [18].

областей фазового расслоения, вызванным влиянием ионов $\mathrm{Er}^{3+}$.

Полярность областей фазового расслоения во всех изученных $R \mathrm{Mn}_{2} \mathrm{O}_{5}$ обусловлена следующими двумя факторами. Внутри этих областей двойной обмен, связанный с переносом $e_{g}$-электронов между парами ионов $\mathrm{Mn}^{3+}-\mathrm{Mn}^{4+}$, приводит к тому, что в позициях ионов $\mathrm{Mn}^{4+}$ (кислородных октаэдрах) оказываются ян-теллеровские ионы $\mathrm{Mn}^{3+}$, которые деформируют эти октаэдры. В свою очередь, меньшие по размеру ионы $\mathrm{Mn}^{4+}$ (по сравнению с ионами $\mathrm{Mn}^{3+}$ ) оказываются в нецентральных пятиугольных пирамидах и также добавочно их искажают. Оба фактора приводят к нецентросимметричности областей фазового расслоения и к их полярности [15-18]. Эти области формируют суперпараэлектрическое состояние, которое ниже некоторых температур находится в замороженном суперпараэлектрическом состоянии. Отклик локальных полярных областей в таком состоянии на приложенное электрическое поле имеет вид петель гистерезиса электрической поляризации с остаточной поляризацией.

Замороженное суперпараэлектрическое состояние для локальных сегнетоэлектрических областей в диэлектрической центросимметричной матрице было рассмотрено ранее теоретически [29], и экспериментально обнаружено в $R \mathrm{Mn}_{2} \mathrm{O}_{5} \quad(R=\mathrm{Gd}, \mathrm{Bi}, \mathrm{Tb}, \mathrm{Er})[15,16,18,19]$. В этих работах было показано, что замороженное суперпараэлектрическое состояние существует до температур, при которых кинетическая энергия свободных носителей заряда сравнивается с высотой барьеров на границах областей. Этот же критерий соответствует и требованию к температурам существования магнитного состояния, что и наблюдается нами в данной работе (сравни вставку к рис. 5, $a$ для осей $a$ и $b$ и рис. 7).
Таким образом, нанообласти фазового расслоения действительно являются мультиферроидными и существуют до температур, значительно превышающих температуры магнитного и сегнетоэлектрического упорядочений матрицы кристаллов.

\section{4. Температурная зависимость теплоемкости ErMO.}

Из магнитных и диэлектрических исследований следует, что в кристаллах ErMO имеются два типа фазовых превращений: магнитный и сегнетоэлектрический фазовые переходы в матрице кристалла; а также фазовые превращения в 2D-областях фазового расслоения. Эти превращения наблюдаются при температурах, до которых существует замороженное суперпарамагнитное и суперпараэлектрическое состояния. Рассмотрим, как эти изменения состояний проявляются в температурной зависимости теплоемкости ErMO. При этом проведем сравнение с ранее изученными теплоемкостями в ЕСМО и ЕMO [7]. На рис 8, a представлены температурные зависимости теплоемкости в ЕСMO и ЕMO [7]. Как отмечалось выше, в ЕСМО ван-флековские ионы $\mathrm{Eu}^{3+}$ (с основным состоянием $\left.\left({ }^{7} F_{0}\right)\right)$ являются слабо магнитными и магнитный момент у них нарастает с ростом температуры только за счет подмешивания возбужденного магнитного состояния ${ }^{7} F_{1}$. Магнитный момент ионов $\mathrm{Eu}^{3+}$ мал и ориентирован в плоскости $a b$ при всех температурах. Намагниченность марганцевой подсистемы в ЕСМО и ЕМО также ориентирована в плоскости $\mathrm{ab}$ и отсутствует поперечное поле, индуцирующее диамагнитный отклик в марганцевой подсистеме. Таким образом, эффективное поле, индуцируемое намагниченностью ионов $\mathrm{Eu}^{3+}$, слабо влияет на состояния марганцевых подсистем этих соединений. Для EMO наблюдаются отчетливые аномалии теплоемкости в окрестностях температур $T_{1}=39 \mathrm{~K}, T_{2}=21 \mathrm{~K}$. Они обусловлены антиферромагнитным и сегнетоэлектрическим фазовыми переходами, соответственно, которые ранее наблюдались в [30].

Температурные зависимости $C_{p} / T$ для ECMO обнаруживают аномалии, сдвинутые в более низкие температуры по сравнению с ЕМО: вблизи $34 \mathrm{~K}$ и размытую аномалию вблизи $15 \mathrm{~K}$ (рис. 8,a), также определяемые последовательностью антиферромагнитных и сегнетоэлектрических фазовых переходов. Таким образом, поведение теплоемкости в ЕСМО и ЕМО при низких температурах $(T<50 \mathrm{~K})$ определяется динамикой как фононной, так и магнитной подсистем [7].

В кристаллах ErMO наблюдается принципиально другая зависимость $C_{p} / T(T)$ (рис. 8,b). В высокотемпературной области (при $T>60 \mathrm{~K}$ ) изменение $C_{p} / T$ линейны с изломом в окрестности $160 \mathrm{~K}$ (левая вставка рис. $8, b)$. В то время как в ЕМО и ЕСМО температурная зависимость теплоемкости $C_{p} / T$ существенно нелинейна при высоких температурах (рис. 8, a). Указанное выше изменение линейного наклона температурной 

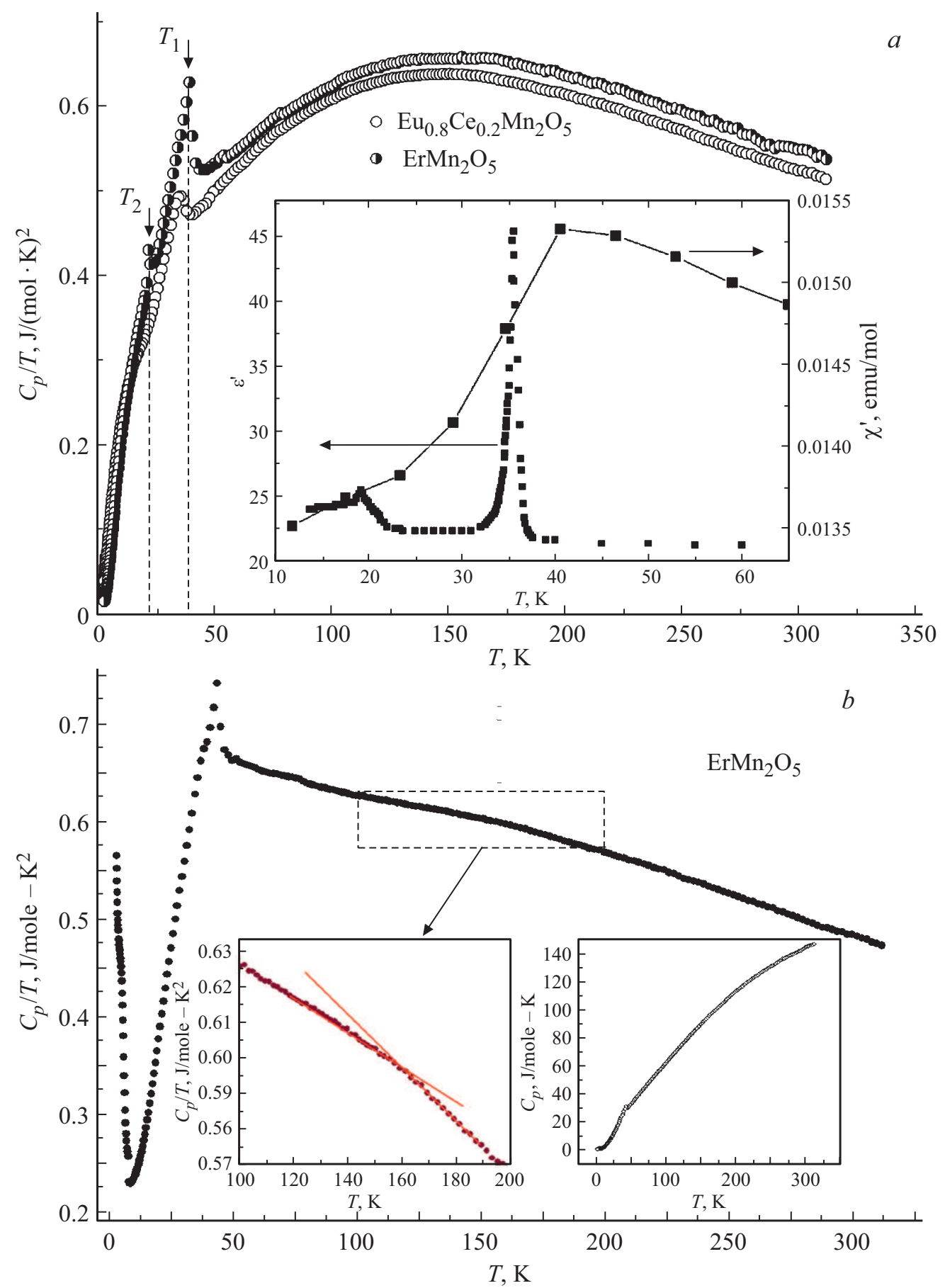

Рис. 8. (a) Температурная зависимость $C_{p} / T$ для ЕCMO и EuMn ${ }_{2} \mathrm{O}_{5}$. На вставке представлены температуры магнитного и сегнетоэлектрического фазовых переходов в $\mathrm{EuMn}_{2} \mathrm{O}_{5}$, которые относятся и к матрице ЕСМО [7]. (b) Температурная зависимость $C_{p} / T$ для ErMO. На левой вставке в увеличенном масштабе представлено поведение $C_{p} / T$ в интервале температур $100-200 \mathrm{~K}$. На правой вставке - зависимость теплоемкости $C_{p}(T)$ во всем диапазоне температур.

зависимости теплоемкости в ErMO коррелирует с температурой исчезновения замороженных суперпарамагнитного и суперпараэлектрического состояний областей фазового расслоения вдоль оси $a$ (левая вставка рис. $8, b$ и рис. 7). В низкотемпературной области зависимости $C_{p} / T$ ErMO обнаруживают две аномалии: узкую, с максимумом при $T_{1}=43 \mathrm{~K}$ и широкую, в виде излома в окрестности $39 \mathrm{~K}$ на фоне резкого падения зависимости $C_{p} / T$ при уменьшении температуры. Указанные температуры аномалий $C_{p} / T(T)$ близки к температурам магнитного упорядочения $T_{\mathrm{N}}=44 \mathrm{~K}$ и сегнетоэлектрического фазового перехода $T_{\mathrm{C}}=39 \mathrm{~K}$ в ErMO.

Существенное отличие поведения теплоемкости ErMO (рис. 8, $b$ ) от ЕСМО (рис. 8, $a$ ) естественно 
связать с влиянием сильномагнитного иона $\mathrm{Er}^{3+}$ с большим орбитальным вкладом на марганцевую подсистему, отсутствующим в ЕСМО.

Особенно существенно проявляются различия влияния ионов $\mathrm{Er}^{3+}$ и $\mathrm{Eu}^{3+}$ в ErMO (рис. 8, $b$ ) и ЕСМО (рис. $8, a$ ), соответственно, на низкотемпературную теплоемкость. Дело в том, что при температурах ниже магнитного и сегнетоэлектрического упорядочений в обоих этих кристаллах имеются фазовые переходы из соразмерных магнитных фаз в несоразмерные фазы, в которых скачком изменяются волновые вектора магнитных структур. Как правило, сегнетоэлектрическое упорядочение реализуется в интервале существования соразмерных магнитных фаз и слабо изменяется при низкотемпературных магнитных соразмерно-несоразмерных фазовых переходах [2]. В ErMO, в отличие от других мультиферроиков $R \mathrm{Mn}_{2} \mathrm{O}_{5}$, имеется аномальная низкотемпературная несоразмерная магнитная фаза LT-1DICM, возникающая ниже $T \sim 8 \mathrm{~K}$, в которой скачком уменьшается электрическая поляризация. Как это отмечалось выше, сегнетоэлектрическая поляризация обменно-стрикционной природы в $R \mathrm{Mn}_{2} \mathrm{O}_{5}$ формируется вдоль оси $b$ за счет обменной стрикции в соразмерной магнитной фазе. Сильно усиливающаяся связь с решеткой ионов $\mathrm{Er}^{3+}$ вдоль оси $c$ кристаллов, резко увеличивает коэрцитивное поле поляризации, обусловленной обменной стрикцией, скачком уменьшая ее величину [2]. Мы связываем резкое нарастание зависимости $C_{p} / T$ при понижении температуры при $T<8 \mathrm{~K}$ (рис. $8, b$ ) с фазовым переходом в LT 1DICM фазу.

\section{4. Заключение}

Проведено комплексное исследование магнитных и сегнетоэлектрических свойств, теплоемкости и СВЧ магнитной динамики мультиферроика $\mathrm{ErMn}_{2} \mathrm{O}_{5}$, в котором сегнетоэлектрическое упорядочение матрицы кристалла индуцируется его магнитным упорядочением. Эти упорядочения возникают при низких, но близких (39 и $44 \mathrm{~K})$ температурах, соответственно, обеспечивая сильную магнитоэлектрическую связь. Характерным свойством $\mathrm{ErMn}_{2} \mathrm{O}_{5}$ является наличие зарядового упорядочения, обусловленного одинаковым количеством ионов $\mathrm{Mn}^{3+}$ и $\mathrm{Mn}^{4+}$, расположенных в соседних слоях, перпендикулярных оси $c$ кристалла. Конечная вероятность туннелирования $e_{g}$ электронов между соседними парами ионов $\mathrm{Mn}^{3+}-\mathrm{Mn}^{4+}$ приводит к энергетической выгодности самопроизвольного формирования нанообластей фазового расслоения. Эти области представляют собой полупроводниковые гетероструктуры, содержащие чередующиеся ферромагнитные слои с различными магнитными моментами, зависящими от числа ферромагнитных пар ионов $\mathrm{Mn}^{3+}-\mathrm{Mn}^{4+}$ в отдельных слоях сверхрешеток - гетероструктур. Формирующиеся за счет самоорганизации области фазового расслоения занимают малый объем матрицы кристалла. Основное динамически равновесное состояние областей фазового расслоения формируются при балансе сильных взаимодействий: двойного обмена (с характерной энергией $0.3 \mathrm{eV})$, взаимодействия Яна-Теллера $(0.7 \mathrm{eV})$ и кулоновского отталкивания $(1 \mathrm{eV})$. По этой причине они существуют в широком диапазоне температур от низких до температур выше комнатной, являясь мультиферроидными и с сильным магнитоэлектрическим взаимодействием. В работе впервые исследовано влияние ионов $\mathrm{Er}^{3+}$, обладающих большим магнитным моментом, с существенным орбитальным вкладом, обеспечивающим сильную связь с решеткой. Показано, что именно влияние ионов $\mathrm{Er}^{3+}$ в $\mathrm{ErMn}_{2} \mathrm{O}_{5}$ обуславливает появление спонтанной электрической поляризации областей фазового расслоения, которая сосуществует с их ферромагнитным упорядочением. Влияние ионов $\mathrm{Er}^{3+}$ на состояния проводящих ферромагнитных областей фазового расслоения приводит к сложной картине температурных изменений намагниченности ErMO, обусловленной самосогласованным влиянием магнитных $\mathrm{Er}$ и $\mathrm{Mn}$ подсистем друг на друга за счет $\mathrm{Er}-\mathrm{Mn}$ обменного взаимодействия. B ErMO обнаружен резкий рост температурной зависимости $\mathrm{Cp} / \mathrm{T}$ при температурах ниже $8 \mathrm{~K}$, обусловленный низкотемпературным, одномерным несоразмерным магнитным фазовым переходом LT 1DICM, резко скачком понижающим обменно-стрикционную электрическую поляризацию. Это обусловлено особенностями основного состояния ионов $\mathrm{Er}^{3+}$.

\section{Конфликт интересов}

Авторы заявляют об отсутствии конфликта интересов.

\section{Список литературы}

[1] N. Hur, S. Park, P.A. Sharma, J.S. Ahn, S. Guba, S.-W. Cheong. Nature (London) 429, 392 (2004).

[2] Y. Noda, H. Kimura, M. Fukunaga, S. Kobayashi, I. Kagomiya, K. Kohn. J. Phys.: Condens. Matter 20, 434206 (2008).

[3] P.G. Radaelli, L.C. Chapon. J. Phys.: Condens. Matter 20, 434213 (2008).

[4] P.G. de Gennes. Phys. Rev. 118, 141 (1960).

[5] Л. П. Горьков. УФН 168, 655 (1998).

[6] J. van den Brink, D.I. Khomskii. J. Phys.: Cond. Matter 20, 434217 (2008).

[7] V.A. Sanina, E.I. Golovenchits, V.G. Zalesskii, S.G. Lushnikov, M.P. Scheglov, S.N. Gvasaliya, A. Savvinov, R.S. Katiyar, H. Kawaji, T. Atake. Phys. Rev. B 80, 224401 (2009).

[8] V.A. Sanina, E.I. Golovenchits, V.G. Zalesskii, M.P. Scheglov. J. Phys.: Condens. Matter 23, 456003 (2011).

[9] Е.И. Головенчиц, В.А. Санина, В.Г. Залесский. Письма в ЖЭТФ 95, 429 (2012).

[10] V.A. Sanina, E.I. Golovenchits, V.G. Zalesskii. J. Phys.: Condens. Matter 24, 346002 (2012).

[11] В.А. Санина, Б.Х. Ханнанов, Е.И. Головенчиц. ФТТ 59, 1932 (2017).

[12] V.A. Sanina, E.I. Golovenchits, V.G. Zalesskii, B.Kh. Khannanov. J. Phys.: Condens. Matter 25, 336001 (2013). 
[13] B.Kh. Khannanov, V.A. Sanina, E.I. Golovenchits. J. Phys. Conf. Ser, 572, 012046 (2014).

[14] V.A. Sanina, E.I. Golovenchits, B.Kh. Khannanov, M.P. Scheglov, V.G. Zalesskii. JETP Lett. 100, 407 (2014).

[15] B.Kh. Khannanov, V.A. Sanina, E.I. Golovenchits, M.P. Scheglov. JETP Lett. 103, 248 (2016).

[16] B.Kh. Khannanov, V.A. Sanina, E.I. Golovenchits, M.P. Scheglov. JMMM 421, 326 (2017)

[17] В.А. Санина, Б.Х. Ханнанов, Е.И. Головенчиц, М.П. Щеглов. ФТТ 60, 531 (2018).

[18] Б.Х. Ханнанов, Е.И. Головенчиц, В.А. Санина. ФТТ 62, 257 (2020).

[19] Б.Х. Ханнанов, Е.И. Головенчиц, В.А. Санина. ФТТ 62, 574 (2020).

[20] С.И. Воробьев, Е.И. Головенчиц, В.Р. Коптев, Е.Н. Комаров, С.А. Котов, В.А. Санина, Г.В. Щербаков. Письма в ЖЭТФ 91, 561 (2010).

[21] С.И. Воробьев, Д.С. Андриевский, С.Г. Барсов, А.Л. Геталов, Е.И. Головенчиц, Е.Н. Комаров, С.А. Котов, А.Э. Морослин, А.Ю. Мищенко, В.А.Санина, Г.В. Щербаков. ЖЭТФ 150, 1170 (2016).

[22] М.Ю. Каган, К.И. Кугель. УФН 171, 533 (2001).

[23] J. Lorenzana, J.C. Castellani, C. Castro Di. Europhys. Lett. 57, 704 (2002).

[24] K.I. Kugel', A.L. Rakhmanov, A.O. Sboychakov, F.V. Kustmarsev, N. Poccia, A. Bianconi. Supercond. Sci. Technol. 22, 014007 (2009).

[25] Е.И. Головенчиц, Б.Х. Ханнанов, В.А. Санина. Письма в ЖЭТФ 111, 826 (1920).

[26] В.А. Санина, Л.М. Сапожникова, Е.И. Головенчиц, Н.В. Морозов. ФТТ 30, 3015 (1988).

[27] А.В. Бабинский, Е.И. Головенчиц, Н.В. Морозов, Л.М. Сапожникова. ФТТ 34, 108 (1992).

[28] A. Maeda, T. Satake, T. Fujimori, H. Kuroda. J. Phys.: Condens. Matter 2, 245 (1990).

[29] M.D. Glinchuk, E.A. Eliseev, A.N. Morozovska. Phys. Rev. B 78, 134107 (2008).

[30] H. Nakamura, M. Ishikawa, K. Kohn, M. Ishikawa, J. Phys. IV France, C 1, 7, 365 (1997).

Редактор Ю.Э. Китаев 